\title{
Numbers needed to treat to prevent tuberculosis
}

To the Editor:

A new action framework advocacy document for tuberculosis elimination in low-incidence countries has recently been published in this journal [1]. We completely agree with the importance of addressing the goal of elimination of tuberculosis, which may be achievable in regions such as Western Europe and North America, but we are concerned that this goal may be overly ambitious at this point in time. As protective immunity can, unfortunately, not be achieved by a vaccine or other immune-based intervention, tuberculosis control still relies on early identification and treatment of active tuberculosis, and preventive chemotherapy of individuals with latent Mycobacterium tuberculosis infection (LTBI). However, both the tuberculin skin test (TST) and interferon- $\gamma$ release assays (IGRAs) (the ELISA-based QuantiFERON-TB Gold In-Tube assay (QIAGEN, Venlo, the Netherlands) or the ELISPOT-based T-SPOT.TB assay (Oxford Immunotec, Oxford, UK)) that are recommended to diagnose LTBI have a low positive predictive value for the future development of tuberculosis, and are therefore insufficient as biomarkers to identify individuals developing active disease. With tuberculosis being a rare event, the vast majority of individuals with positive test results will never develop disease [2]. Moreover, in immunocompromised individuals, especially in patients with HIV infection, the negative predictive value of an immunodiagnostic test is insufficient to rule out the future development of tuberculosis, as was recently demonstrated in a large study in Europe [3]. Thus, systematic testing and treatment of LTBI of risk groups [1] is considered difficult to implement.

In order to optimise allocation of the available resources for tuberculosis elimination, the first step would be an improvement in the knowledge of risk groups for tuberculosis in low-incidence countries. More recently, it has become evident that the risk of the development of tuberculosis among patient groups classically considered at risk is highly variable, and is not necessarily associated with the results of immunodiagnostic testing by the TST or IGRAs [2-5]. More detailed knowledge of groups at actual risk of tuberculosis and on immunodiagnostic test performance in these risk groups may provide better guidance for physicians on priorities for targeting tuberculosis testing and LTBI treatment in low-incidence countries.

The decision to administer prophylactic treatment to a certain risk group should depend on the estimated number needed to treat to prevent one case of tuberculosis. Although not selected by a systematic approach, we made use of the fact that several studies with large sample sizes from low-incidence countries of tuberculosis were recently published from three major groups classically considered at risk for progression. In these studies, tuberculosis contacts [2, 4, 5], immunocompromised hosts [3] and healthcare workers [6-8] were evaluated for LTBI and subsequent progression. We have calculated the number needed to treat to prevent one case of tuberculosis (table 1), and found that the number needed to treat was very similar across three studies evaluating 1579, 5020 and 4774 tuberculosis contacts, respectively, with a positive TST or IGRA, ranging from 30 to 37 [2, 4, 5] (table 1). In immunocompromised individuals with a positive test $(\mathrm{n}=1537)$ [3], the number needed to treat to prevent a case was higher and ranged from approximately 50 to 80 (table 1). Notably, as incident cases of tuberculosis were mainly HIV positive, the number of HIV-infected persons with a positive test needed to treat was lower (range 14.0-25.5) and tuberculosis cases were only found in patients with ongoing viral replication [3]. It should be emphasised that incident tuberculosis cases also occurred in individuals with negative test results [2,3,5], especially among patients with HIV infection. In patients with chronic renal failure $(n=270)$ or rheumatoid arthritis $(n=199)$, stem cell $(n=103)$ or solid organ transplant recipients $(n=197)$ [3], and healthcare workers $(n>15000)$ [6-8], the number of individuals with LTBI needed to treat to prevent one case could not be calculated because no tuberculosis cases occurred during 2 years of follow up in these cohorts

In the absence of better biomarkers [9], comparing the number needed to treat to prevent one case is helpful to define groups with highest risk of developing tuberculosis and to prioritise existing prevention strategies. These strategies should particularly focus on HIV-infected individuals, preferentially with ongoing viral replication, and on close contacts of infectious cases of tuberculosis, although higher numbers are needed to screen and treat among contacts. In contrast, given the low number or even absence of incident cases in other groups classically considered at risk, screening approaches more closely targeted to individuals with additional risk factors would seem more effective to identify candidates who will most likely benefit from preventive chemotherapy [10]. Finally, some groups formerly considered at 


\begin{tabular}{|c|c|c|c|c|}
\hline Population & First author [ref.] & Subpopulations & NNT & Test \\
\hline \multirow[t]{5}{*}{ Contacts } & ZELLWEger [2] & & 37.0 & ELISA \\
\hline & & & 36.5 & ELISPOT \\
\hline & GEIS [4] & & 34.5 & ELISA \\
\hline & SŁoot [5] & All & 88.9 & ELISA \\
\hline & & Close contacts (first circle) & 30.4 & $\begin{array}{c}\text { and/or } \\
\text { TST }\end{array}$ \\
\hline \multirow[t]{6}{*}{ Immunocompromised patients } & Sester [3] & HIV, CRF, SOT, SCT, RA & 49.7 & TST \\
\hline & & & 79.5 & ELISA \\
\hline & & & 64.3 & ELISPOT \\
\hline & & HIV & 14.0 & TST \\
\hline & & & 25.5 & ELISA \\
\hline & & & 21.3 & ELISPOT \\
\hline
\end{tabular}

The low number of TB cases present in each of the studies precludes the calculation of meaningful confidence intervals around the NNT. TST: tuberculin skin test; CRF: chronic renal failure; SOT: solid organ transplantation; SCT: stem cell transplantation; RA: rheumatoid arthritis.

risk of tuberculosis (e.g. healthcare workers) are no longer risk groups in low-incidence countries [6-8], unless having had substantial contact to a patient with tuberculosis. It should be stated that given the inadequate test characteristics of the current diagnostics, not all tuberculosis will be prevented by these strategies, since active disease will continue to occur in those tested negative.

@ERSpublications

Comparing the NNT to prevent one case can help define groups at highest TB risk and prioritise prevention strategies http://ow.ly/TITzR

Martina Sester ${ }^{1}$, Reinout van Crevel ${ }^{2}$, Frank van Leth ${ }^{3}$ and Christoph Lange ${ }^{4,5,6,7,8}$

${ }^{1}$ Dept of Transplant and Infection Immunology, Saarland University, Homburg, Germany. ${ }^{2}$ Dept of Medicine, Radboud University Medical Center, Nijmegen, The Netherlands. ${ }^{3}$ Dept of Global Health, Academic Medical Center, University of Amsterdam and Amsterdam Institute for Global Health and Development, Amsterdam, The Netherlands. ${ }^{4}$ Division of Clinical Infectious Diseases, Research Center Borstel, Borstel, Germany. ${ }^{5}$ German Center for Infection Research, Tuberculosis Unit, Borstel, Germany. ${ }^{6}$ International Health/Infectious Diseases, University of Lübeck, Lübeck, Germany. ${ }^{7}$ Dept of Medicine, Karolinska Institute, Stockholm, Sweden. ${ }^{8}$ Dept of Medicine, University of Namibia School of Medicine, Windhoek, Namibia.

Correspondence: Martina Sester, Dept of Transplant and Infection Immunology, Saarland University, Kirrberger Straße, Homburg 66421, Germany. E-mail: martina.sester@uks.eu

Received: June 082015 | Accepted: July 022015

Conflict of Interest: Disclosures can be found alongside the online version of this article at erj.ersjournals.com

\section{References}

1 Lönnroth K, Migliori GB, Abubakar I, et al. Towards tuberculosis elimination: an action framework for low-incidence countries. Eur Respir J 2015; 45: 928-952.

2 Zellweger JP, Sotgiu G, Block M, et al. Risk assessment of tuberculosis in contacts by IFN-gamma release assays. A tuberculosis network european trials group study. Am J Respir Crit Care Med 2015; 191: 1176-1184.

3 Sester M, van Leth F, Bruchfeld J, et al. Risk assessment of tuberculosis in immunocompromised patients. A TBNET study. Am J Respir Crit Care Med 2014; 190: 1168-1176.

4 Geis S, Bettge-Weller G, Goetsch U, et al. How can we achieve better prevention of progression to tuberculosis among contacts? Eur Respir J 2013; 42: 1743-1746.

5 Sloot R, Schim van der Loeff MF, Kouw PM, et al. Risk of tuberculosis after recent exposure. A 10-year follow-up study of contacts in Amsterdam. Am J Respir Crit Care Med 2014; 190: 1044-1052.

6 Schablon A, Nienhaus A, Ringshausen FC, et al. Occupational screening for tuberculosis and the use of a borderline zone for interpretation of the IGRA in German healthcare workers. PLoS One 2014; 9: e115322.

7 Dorman SE, Belknap R, Graviss EA, et al. Interferon-gamma release assays and tuberculin skin testing for diagnosis of latent tuberculosis infection in healthcare workers in the United States. Am J Respir Crit Care Med 2014; 189: 77-87.

8 Slater ML, Welland G, Pai M, et al. Challenges with QuantiFERON-TB Gold assay for large-scale, routine screening of U.S. healthcare workers. Am J Respir Crit Care Med 2013; 188: 1005-1010.

9 Chegou NN, Heyckendorf J, Walzl G, et al. Beyond the IFN- $\gamma$ horizon: biomarkers for immunodiagnosis of infection with Mycobacterium tuberculosis. Eur Respir J 2014; 43: 1472-1486. 
From the authors:

We thank Martina Sester and colleagues for their careful reading of our paper [1] and for their support to the prospect of tuberculosis (TB) elimination in low-incidence countries in a foreseeable future. While we respect the views of the authors that the targets may appear too ambitious, we believe that, if the scientific community stay focussed on innovative approaches that can translate into scalable and effective interventions, we could reap the benefits of such interventions within the space of the next two decades [2]. Figure 1 shows the trend in $\mathrm{TB}$ incidence into the future, should current efforts to control $\mathrm{TB}$ - including the treatment of latent Mycobacterium tuberculosis infection (LTBI) - be intensified and boosted by new techniques and approaches.

When discussing the importance of preventive chemotherapy for LTBI in the context of TB elimination, the authors point out two critical issues: the limitations of diagnostic tests for LTBI, and the importance of appropriately defining target populations for systematic LTBI testing. To provide guidance in this area, the World Health Organization (WHO) has recently issued guidelines on LTBI management [4].

Both tuberculin skin testing (TST) and interferon- $\gamma$ release assays (IGRAs) have a poor positive predictive value to determine which individuals with presumed latent infection are more likely to develop active TB, implying that a high proportion of treated individuals would receive medication unnecessarily. Nonetheless, these tests still have a role in helping clinicians improve the health outcomes of their patients and to progress towards TB elimination. Conversely, the negative predictive value of TST and IGRAs is high enough to exclude most individuals who would not benefit from LTBI treatment [5]. Contrary to what Martina Sester and colleagues maintain, the number of active TB cases that will continue to occur among those testing negative is thus unlikely to have a significant public health impact.

We agree with Martina Sester and colleagues that to optimise the allocation of available resources, appropriate selection of risk groups for systematic LTBI testing and treatment is crucial. WHO recommends that individuals with a positive TST or IGRA should be treated for LTBI if they have the following risks: HIV infection, are contacts of pulmonary TB cases, initiate anti-tumour necrosis factor treatment, receive dialysis, prepare for transplantation, or have silicosis. The WHO guidelines group composed of leading world experts using the GRADE (Grading of Recommendations Assessment, Development and Evaluation) approach [6] - made a strong recommendation for LTBI treatment in these groups, on the premise of both direct and surrogate evidence for an increased risk of progression from infection to disease when compared with the general population, and much less upon a positive LTBI test. Martina Sester and colleagues argue that the number of individuals who need to take prophylactic medication in order to prevent one active case (number needed to treat (NNT)) would have been a better outcome measure to select at-risk populations. While this appears to be a logical approach, it also has

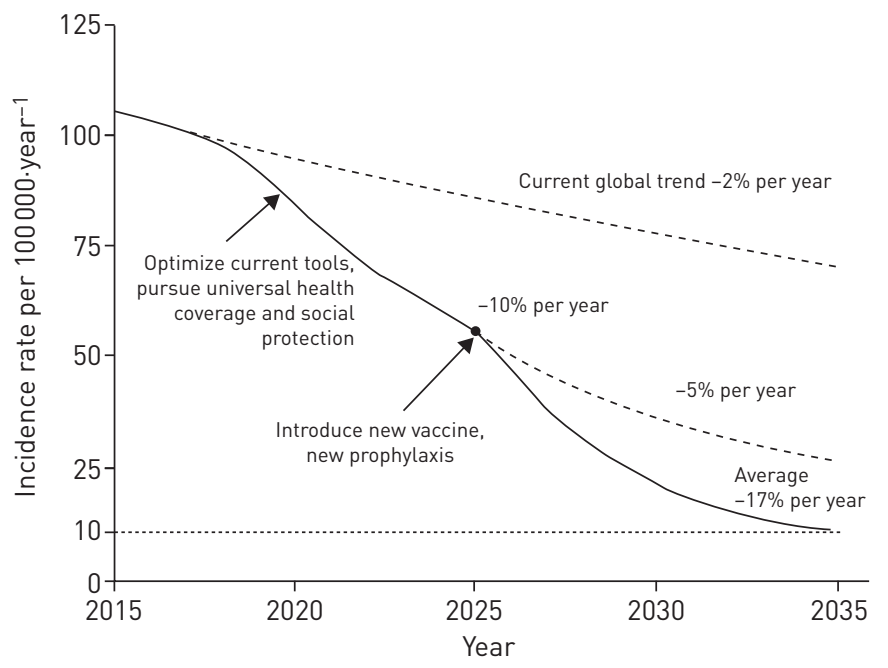

FIGURE 1 Projected trend in the decline of global tuberculosis incidence, from 110 cases per 100000 in 2015 to 10 cases per 100000 or less by 2035. Reproduced from [3] with permission from the publisher. 